\title{
ACE Genotype Distributions Differ between Sporadic and Familial Hypertrophic Cardiomyopathy
}

$\underline{\operatorname{Ara~Kassarjian}^{*}}{ }^{*}$ B.S., and Eleanor Elstein ${ }^{Y}$, M.D., F.R.C.P. (C)

* Faculty of Medicine, McGill University, 3655 Drummond St., Montreal, QC, Canada H3G 1Y6

Ý To whom correspondence should be addressed: Department of Medicine, Division of Cardiology, Royal Victoria Hospital, McGill University, 687 Pine Ave. West, Montreal, QC, Canada H3A 1A1

\section{ABSTRACT}

This study investigated the frequency of ACE genotypes in sporadic hypertrophic cardiomyopathy (HCM) and compared these frequencies to those found in the general population and in familial HCM. Delineation of the genotype of a $287 \mathrm{bp}$ fragment in the ACE gene of 10 patients with confirmed sporadic HCM demonstrated that $2(20 \%)$ were of the DD genotype, $5(50 \%)$ of the ID genotype, and 3 $(30 \%)$ of the II genotype. These genotype distributions did not differ significantly from controls $(p<$ 0.57). Comparison of the present results with genotype frequencies in familial HCM reported in prior studies revealed a significant difference in genotype distribution between sporadic and familial HCM $(p<0.04)$. These findings indicate that the frequency of the ACE genotype does not appear to differ between patients with sporadic HCM and the general population. However, the results suggest that, with regard to the ACE polymorphism studied, genetic differences may exist between the sporadic and familial forms of HCM.

\section{INTRODUCTION}

Over the past decade, much interest has developed regarding the role of the renin-angiotensin system in cardiovascular disease. Specifically, the angiotensin-I converting enzyme (ACE), the enzyme responsible for converting angiotensin I to angiotensin II, has been the focus of a considerable number of investigations due to its apparent widespread effects on multiple organ systems, including the heart, the vascular system, and the kidneys. Angiotensin has been shown to have vasoconstrictor activity on vascular endothelium and may thus play a role in the pathophysiology of hypertension. Also, angiotensin has been shown to stimulate smooth muscle hypertrophy and hyperplasia through the release of growth factors, such as platelet derived growth factor, and through the activation of proto-oncogenes (1). Recent advances in molecular biology have localized the ACE gene to chromosome 17 (2). Further analysis demonstrated a polymorphic region consisting of the presence or absence (insertion/deletion) of a $287 \mathrm{bp}$ fragment (3). 
Many studies have attempted to determine whether specific ACE genotypes are associated with certain traits or diseases. For instance, Rigat et al. found that the insertion/deletion polymorphism accounted for nearly half the variance of serum ACE levels, with deletion homozygotes (DD genotype) having significantly higher serum ACE levels (4). Subsequently, Cambien et al. demonstrated that the DD genotype is more frequent in patients who had a myocardial infarction (MI) and thus constitutes a risk factor for MI, particularly in those patients considered to be at low risk (i.e. without hypertension, high plasma lipid levels, or smoking history) (5). Further analysis revealed that, in patients with previous MI, there was increased frequency of the DD genotype among those with a parental history of MI. This suggested that genetic variation at the ACE locus may be involved in the risk for MI (6).

In another study, Raynolds et al. found an increased frequency of the DD genotype in patients with ischemic or dilated cardiomyopathy, indicating that the ACE DD genotype may be a risk factor for heart failure associated with these types of cardiomyopathy (7). An increased frequency of the DD genotype has also been associated with left ventricular hypertrophy (LVH) (8), coronary artery disease (9), familial hypertrophic cardiomyopathy (HCM), and a family history of sudden cardiac death (SCD) (10). In addition, the DD genotype has been found to influence the phenotypic expression of left ventricular hypertrophy in HCM (11).

Considering the numerous associations of the ACE DD genotype to cardiac disease, the purpose of this investigation was to delineate the frequency of the DD genotype in sporadic HCM and then compare these findings to the frequency of the DD genotype in familial HCM and in the general population.

\section{METHODS}

\section{Selection of Subjects and Controls}

Blood samples were taken from 10 patients with sporadic HCM. The diagnosis was confirmed with echocardiography and a detailed family history. The control group consisted of 25 patients with no documented evidence of cardiac disease who had been randomly selected from an unrelated unpublished study.

Informed consent for inclusion in this study was obtained from all patients.

\section{Isolation of DNA}

DNA was isolated from peripheral lymphocytes as previously described (12).

\section{Amplification}

Polymerase chain reaction was performed using 1.0-1.7 $\mu \mathrm{g}$ of template DNA and 25 pmol each of DCP-sense (5'CTGGAGACCACTCCCATCCTTTCT 3') and DCP-anti-sense (5'GATGTGGCCATCACATTCGTCAGAT 3') primers. These were prepared in a final volume of $50 \mu 1$ of buffer solution, containing $1.5 \mathrm{mM} \mathrm{MgCl} 2,50 \mathrm{mM} \mathrm{KCl}, 10 \mathrm{mM}$ Tris- $\mathrm{HCl} \mathrm{pH}$ 9.0, 0.5 mM of each dNTP, and 5 units of Taq polymerase (Pharmacia). The DNA was amplified for 28 cycles with denaturation at $955 \mathrm{C}$ for 1 min, annealing at 55šC for $2 \mathrm{~min}$, and extension at $72 \check{\mathrm{s} C} \mathrm{C}$ for $3 \mathrm{~min}$, with a final extension at $72 \check{\mathrm{s}} \mathrm{C}$ for $10 \mathrm{~min}$ using a thermocycler (model PTC-150, MJ Research, Watertown, Massachusetts, USA). The PCR products were visualized by electrophoresis on a $2 \%$ agarose gel stained with ethidium bromide.

\section{RESULTS}

The results of gel electrophoresis on PCR products of DNA that was extracted from peripheral lymphocytes of subjects having the DD genotype revealed a 190-bp fragment, whereas subjects with the II genotype demonstrated a 490-bp fragment (Fig. 1). Heterozygotes for the ACE ID polymorphism produced both bands 
as well as an intermediate third band that represents a heteroduplex DNA fragment described previously (4).

Analysis of the results of electrophoresis from 10 patients with sporadic HCM demonstrated that 2 (20\%) were of the DD genotype, $5(50 \%)$ of the ID genotype, and $3(30 \%)$ of the II genotype, yielding an allele frequency of 0.45 for the D allele and 0.55 for the I allele (Table 1). The 25 control subjects showed 4 (16\%) DD, 17 (68\%) ID, and 4 (16\%) II genotypes, yielding an allele frequency of 0.50 for the D allele and 0.50 for the I allele.

Statistical analysis revealed that the ACE genotype frequencies were not significantly different between the patients with sporadic HCM and controls $(p<0.57)$. Further comparison of patients from this study with historical controls (Cambian et al., Marian et al., and Schunkert et al.) $(5,8,10)$ failed to show a significant difference in ACE genotype distribution ( $p<0.69, p<0.58$, and $p<0.57$, respectively). In addition, the ACE genotype distribution in patients from this study was not significantly different from the distribution in sporadic HCM patients reported by Lechin et al. (11) $(p<0.69)$ (Table 2).

The distribution of ACE genotypes in sporadic HCM patients in this study was significantly different $(p<$ 0.04) from the ACE genotype distributions in familial HCM reported by Marian et al. This difference was more significant $(p<0.006)$ when only patients with familial HCM and a family history of sudden cardiac death (SCD) were included (Table 3).

\section{DISCUSSION}

The ACE genotypes of 10 patients with sporadic HCM have been determined in the present study, and the distribution of these genotypes is similar to that reported by another author (11). No significant difference was found in the distribution of the ACE genotypes between sporadic HCM and a control population. Comparison of these results with ACE genotype distributions found in control populations from other studies of ACE polymorphisms also showed no significant difference $(5,8,10)$. These findings suggest that a similar distribution of ACE genotypes exists between patients with sporadic HCM and the general population.

Previous literature has described the distribution of ACE polymorphisms in HCM subgroups (10). Data from these studies demonstrated an increased frequency of the DD genotype in familial HCM as compared to a control population. However, the present study does not support an increased frequency of the DD genotype in the sporadic form of HCM. Although patients with sporadic HCM did not show a higher frequency of DD genotype than controls, the frequency of this genotype (and thus of the D allele) in sporadic HCM patients was significantly lower than that reported elsewhere for familial HCM patients.

The implications of the difference in DD genotype frequency between sporadic and familial HCM patients are not entirely clear. Despite the influence of the DD genotype on LVH shown by Lechin et al., it is possible that the ACE gene polymorphism has no influence on the phenotypic expression of HCM in the sporadic form. It must be noted that neither the present study of sporadic HCM patients nor the study of familial HCM patients by Marian et al. was controlled for LVH severity. Because a large proportion of the familial HCM patients in the study by Marian et al. had a familial history of SCD, the increased frequency of the DD genotype in these patients may actually represent the association between increased DD genotype frequency and severity of $\mathrm{LVH}$, and not an association between increased DD frequency and familial HCM. It remains possible that the difference in DD genotype frequencies between familial and sporadic HCM reported here is not truly a function of the form of the disease, but rather reflects a difference in LVH severity between the two populations studied.

It should be noted that the frequency of the DD genotype reported for the HCM populations in prior studies may be over-represented, whereas it is unlikely that a similar overestimation exists in the present study. While the subjects in the present study represented totally unrelated cases of HCM, the $100 \mathrm{HCM}$ patients in 
the study by Marian et al. spanned merely 18 families, which may have skewed the sample population toward certain familial genotypes.

The possibility of mistyping ACE gene heterozygotes, as reported by Shanmugan et al (13), must also be considered. Although mistyping of heterozygotes (ID) as deletion homozygotes (DD) would falsely increase the frequency of the DD genotype, cases and controls within a given study would be affected to the same degree, and thus the validity of associations found between genotype and disease would be preserved. However, variability in the degree of mistyping among different studies may compromise the validity of comparing numerical results from different studies, as performed here. In order to minimize this possibility of mistyping, an excess ( $25 \mathrm{pmol}$, as opposed to the standard $10 \mathrm{pmol}$, per $50 \mu \mathrm{l}$ ) of each primer was used in the present study, as suggested by Perna et al. (14). This method of using excess primer was not employed in other studies investigating the association of the DD genotype with HCM.

In conclusion, this study demonstrates no significant difference in the distribution of the ACE gene polymorphisms in sporadic HCM compared to the general population. When results from this study were compared to the literature on familial HCM, there was no increased frequency of the DD genotype in sporadic HCM. Although confirmation by studies of larger populations is necessary, this finding suggests that, with regard to the ACE polymorphism studied, genetic differences may exist between the sporadic and familial forms of HCM.

\section{ACKNOWLEDGMENTS}

This work was conducted under a Medical Research Council of Canada Student Research Award. Financial support was also provided by the Department of Medicine of the Royal Victoria Hospital. The authors also wish to thank Patrice Vaillancourt for his technical assistance.

\section{REFERENCES}

1. Baker KM, Booz GW, Dostal DE. Cardiac actions of angiotensin II: role of an intracardiac renin angiotensin system. Annual Review of Physiology 54: 227-41; 1992.

2. Soubrier F, Alhenc-Gelas F, Hubert C, et al. Two putative active centers in human angiotensin I converting enzyme revealed by molecular cloning. Proceedings of the National Academy of Sciences of the United States of America 85(24): 9386-90; 1988.

3. Rigat B, Hubert C, Alhenc-Gelas F, et al. An insertion/deletion polymorphism in the angiotensin Iconverting enzyme accounting for half the variance of serum enzyme levels. Journal of Clinical Investigation 86(4): 1343-46; 1990.

4. Rigat B, Hubert C, Corvol P, et al. PCR detection of the insertion/deletion polymorphism of the human angiotensin converting enzyme gene (DCP1) (dipeptidyl carboxy-

peptidase 1). Nucleic Acids Research 20(6): 1433; 1992.

5. Cambien F, Poirier O, Lecerf L, et al. Deletion polymorphism in the gene for angiotensin-converting enzyme is a potent risk factor for myocardial infarction. Nature 359(6396): 641-44; 1992.

6. Tiret L, Kee F, Poirier O, et al. Deletion polymorphism in angiotensin-converting enzyme gene associated with parental history of myocardial infarction. Lancet 341(8851): 991-2; 1993.

7. Raynolds MV, Bristow MR, Bush EW, et al. Angiotensin-converting enzyme DD genotype in patients with ischaemic or idiopathic dilated cardiomyopathy. Lancet 342(8879): 1073-75; 1993. 
8. Schunkert H, Hense HW, Holmer SR, et al. Association between a deletion polymorphism of the angiotensin-converting-enzyme and left ventricular hypertrophy. New England Journal of Medicine 360(23): 1634-8; 1994.

9. Beohar N, Damaraju S, Prather A, et al. Angiotensin-I converting enzyme genotype DD is a risk factor for coronary artery disease. Journal of Investigative Medicine 43(3): 275-80; 1995.

10. Marian AJ, Yu QT, Workman R, et al. Angiotensin-converting enzyme polymorphism in hypertrophic cardiomyopathy and sudden cardiac death. Lancet 342(8879): 1085-6; 1993.

11. Lechin M, Quinones MA, Omran A, et al. Angiotensin-I converting enzyme genotypes and left ventricular hypertrophy in patients with hypertrophic cardiomyopathy. Circulation 92(7): 1808-12; 1995.

12. Leadon SA, Cerutti PA. A rapid and mild procedure for the isolation of DNA from mammalian cells. Analytical Biochemistry 120(2): 282-8; 1982.

13. Shanmugam V, Sell KW, Saha BK. Mistyping ACE heterozygotes. PCR Methods \& Applications 3(2): 120-1; 1993.

14. Perna NT, Batzer MA, Deininger PL, et al. Alu insertion polymorphism: a new type of marker for human population studies. Human Biology 64(5): 641-8; 1992.

\section{BIOGRAPHY}

Ara Kassarjian received a B.S. in Kinesiology/Physiological Science from the University of California at Los Angeles (Los Angeles, CA, USA) in 1992. He is currently a fourth year medical student at McGill University (Montreal, Quebec, Canada). His research on the genetics of hypertrophic cardiomyopathy was conducted during his second year of medical school.

Copyright (C) 1996 by MJM 